\title{
Development of a systematic and practical methodology for the design of vehicles semi-active suspension control system
}

\author{
Hamidreza Bolandhemmat ${ }^{\mathrm{a}}$, Christopher M. Clark ${ }^{\mathrm{b}}$ and Farid Golnaraghi ${ }^{\mathrm{c}}$ \\ ${ }^{3}$ Mechanical and Mechatronics Engineering Department, University of Waterloo, ON, Canada; \\ ${ }^{b}$ Computer Science Department, California Polytechnic State University, CA, USA; ' Mechatronics \\ Systems Engineering, Simon Fraser University, Surrey, BC, Canada
}

\begin{abstract}
In this paper, a novel systematic and practical methodology is presented for design of vehicle semiactive suspension systems. Typically, the semi-active control strategies developed to improve vehicle ride comfort and stability have a switching nature. This makes the design of the controlled suspension systems difficult and highly dependent on an extensive trial-and-error process. The proposed methodology maps the discontinuous control system model to a continuous linear region, where all the time and frequency design techniques, established in the conventional control system theory, can be applied. If the semi-active control system is designed to satisfy some ride and stability requirements, an inverse mapping offers the ultimate control law. At the end, the entire design procedure is summarised in six steps. The effectiveness of the proposed methodology in the design of a semi-active suspension system for a Cadillac SRX 2005 is demonstrated with road tests results. Real-time experiments confirm that the use of the newly developed systematic design method reduces the required time and effort in real industrial problems.
\end{abstract}

Keywords: semi-active suspension systems; Fuzzy systems; nonlinear separation theorem; Skyhook control policy; systematic approach; sub-optimal Skyhook strategy; road test verification

\section{Introduction}

This paper demonstrates a novel practical methodology to design and implement vehicle semi-active suspension systems by using simple analytical models. The research groups at the University of Waterloo and Simon Fraser University investigate a semi-active suspension system in a Cadillac SRX 2005 model equipped with Delphi magnetorheological (MR) semiactive dampers. To illustrate the efficiency of the proposed method, the Cadillac suspension controller is bypassed and replaced with the newly developed control system.

Semi-active suspension systems utilise dampers that can change their damping and stiffness properties, given a low-power electrical signal, and exhibit high-performance vibration isolation. Usually, the command signal is provided by the vehicle computer, or preferably, a low cost micro-controller module. There are two classes of semi-active dampers in practice. 
One is a modification of passive dampers that are equipped with external solenoid valves to vary the oil flow rate between the compression chamber and the reverse chamber of the damper. For the second class, the dampers (for example, MR dampers) change the properties of the material inside the shock to provide different levels of resisting forces.

Sensors are essential components of vehicles semi-active suspension systems. They measure the chassis and wheels motion, relative to the road. The information is then fed back to the vehicle computer, preferably a micro-processor, which in turn, sends a control command to the semi-active dampers. It is, therefore, of great practical interest to develop control strategies that are efficient for embedded programming so that the micro-processor cost and size are reduced.

Also, various control strategies have been proposed to determine the desired damping force. The Skyhook control strategy, introduced by Karnopp et al. [1], is undoubtedly the most widely used control policy for semi-active suspension systems. Following the Skyhook policy, the semi-active damper that mounted between the sprung mass and a stationary sky, emulates a fictitious damper behaviour. It has been established that the Skyhook strategy can significantly attenuate the resonant peak of the sprung mass, enhancing the vehicle's ride comfort.

However, the Skyhook strategy does not address the issues associated with the wheel vibration. As a result, the technique cannot decrease the resonant peak that corresponds to the unsprung mass; offering less improvement in the handling performance and stability of the vehicle. To overcome the disadvantage of the original Skyhook concept, Novak and Valasek [2] have added another fictitious damper between the unsprung mass and the ground. This increases the traction between the vehicle tyre and the ground to enhance the handling characteristics of the vehicle.

Both the original Skyhook strategy and its modified version, the Skyhook-Groundhook control called a hybrid control, are also effective in terms of the simplicity of the control algorithm. Their other advantage is that aside from the information regarding the vehicle's shock characteristics, the versions do not require any a priori knowledge about the dynamics of the vehicle; that is, they are not model-based.

The Skyhook policy can be applied either as a bang-bang controller or in a continuous manner by utilising the sprung mass vertical velocity feedback (however, this version is also discontinuous). The on-off Skyhook controller is usually simpler and better suited for the industrial applications. The control law can be described simply as

$$
c_{\text {sky }}=\begin{array}{ll}
l_{\text {max }} & \text { if } v_{\mathrm{r}} v_{\mathrm{b}} \geq 0 \\
l_{\text {min }} & \text { otherwise }
\end{array} \text {, }
$$

in which $v_{b}$ is the absolute velocity of the sprung mass, and $v_{r}$ the relative velocity between the sprung and unsprung mass across the suspension. Extensive theoretical and experimental studies of the performances of different types of semi-active Skyhook(-Groundhook) controllers can be found in the literature [3-9].

Nevertheless, the controller upper and lower gains; that is $c_{\max }$ and $c_{\min }$ are usually determined by trial-and-error, and there is no systematic method to adjust them. This would make the controllable suspension system development process time consuming, and sometimes too difficult. Consequently, this paper introduces a new methodology that allows for the systematic design and implementation of the on-off Skyhook control strategy for semi-active suspensions.

The discontinuous nature of semi-active control strategies, including the Skyhook policy, is the principle barrier in methodical design. The first step of the new methodology is to apply the Fuzzy system theory to create a network with continuous-valued outputs to emulate the discontinuous controller law. Once the original control strategy is converted to a continuous 
form, different well-established frequency or time domain techniques can be employed to design and adjust suspension system controller parameters.

However, there still remains another challenge, associated with the Skyhook strategy implementation. The semi-active control algorithm requires the absolute velocity of the sprung mass, which is intricate to measure in practice. A common technique to obtain the velocity information is to integrate signals from the accelerometers, attached to the ends of each shock. Theoretically, a single integration of the sensor signal, which has already lost its DC offset by the use of a high-pass filter, yields the required velocity data. However, the high-rate drift of low-cost automotive grade sensors (with typically more than $0.05 \mathrm{~g}$ bias stability and between 50-1000 $\mu \mathrm{g} / \mathrm{Hz}$ noise level) makes this extremely challenging in the real world. An additional goal of this research is to come up with a modified version of the Skyhook control policy, which is independent of the absolute velocity state, while maintaining the performance of the original strategy.

The remainder of the paper is organised in six sections. First, in Section 2, the structure of a general semi-active suspension system is presented and a proper analytical model is assigned to each element of the closed-loop control system. The new design methodology is introduced in Section 3. The real-time implementation results and the corresponding analyses are given in the fourth Section. Finally, the modified Skyhook version called a sub-optimal Skyhook policy is introduced in Section 5. The results of the road tests, showing efficiency of the sub-optimal Skyhook controller, are also presented in the same section.

\section{Structure of the closed-loop semi-active control system}

Figure 1 shows a typical configuration of vehicles semi-active suspension systems. Vector 2 contains the outputs of the sensors, strategically distributed throughout the vehicle, to capture the vehicle's motions. The sensing system can consist of accelerometers, gyroscopes, and displacement sensors with different configurations. The information sent by the sensors is processed by either an estimator, for example an extended Kalman filter (EKF) or ordinary low/ high-pass digital filters. The outputs of the filtering unit are the vehicle states required for the semi-active controller. Frequently used state variables include the absolute vertical velocity of the vehicle body at each corner (shock ends), the absolute velocity of the wheel hubs, each shock deflection and its rate and the vehicle's centre of gravity (CG) kinematics. Incorporating the vehicle states (in this case, the deflection rate of each shock, as well as the absolute velocity of the body corners), the Skyhook control strategy determines the damping characteristic suitable for the current time-step. The control command is accordingly fulfilled by the vehicle semi-active shocks.

The vehicle system and its various suspension components are very complex. Hence, the effort is usually focussed on developing simple analytical models of the vehicle and the suspension system. Then, these models are verified and fine-tuned experimentally. The semi-active

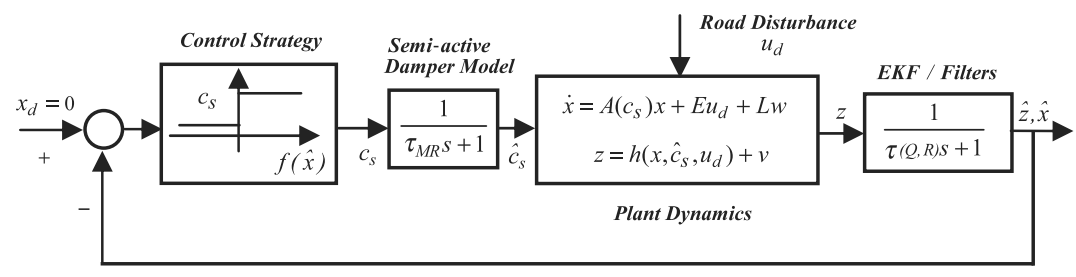

Figure 1. Block diagram of the vehicles semi-active suspension control system. 
dampers behave similarly to low-pass filters with relatively high bandwidths. The bandwidth corresponding to the MR dampers, can be experimentally determined [10,11] as approximately $65 \mathrm{rad} / \mathrm{sec}$. The vehicle dynamics can be expressed by a linear time-varying formulation with respect to the semi-active damping coefficient, $c_{s}$, (which is equal to the $c_{\text {sky }}$ in this study) of the following form:

$$
x^{*}(t)=A\left(c_{s}\right) x+E\left(c_{s}\right) u_{d}+L w^{\prime}
$$

where $x(t)$ and $U_{d}$ refer to the vehicle state vector and inputs to the wheels by the road roughness, respectively. In addition, $A$ and $E$ are the corresponding state and road input matrices. The eigenvalues of the state matrix $A$ match the resonance frequencies of the vehicle body and those of the wheels. The typical values are between 1 and $3 \mathrm{~Hz}$ for the body (roll, pitch, and heave mode) and 8 to $12 \mathrm{~Hz}$ for the wheels.

The validity of the model is verified in the 4-posters test facility as depicted in Figure 2, where the fully instrumented vehicle is excited by sweeping frequency base excitations. Figure 3 portrays the frequency content of a typical input exerted by the base shakers. The road input exhibits its maximum power in frequencies ranging from around 5 to $35 \mathrm{~Hz}$. Accordingly, the primary frequency components of the shakers input are located in the same frequency range to excite the same mode shapes of the vehicle, which are excited by real road disturbances. The frequency response of the test vehicle is then compared with the spectrum, predicted by the analytical model. Consequently, the parameters of the model are modified to approximate the behaviour of the actual vehicle more precisely.

For instance, Figure 4 plots the frequency content of the accelerometer signal, mounted on the front left corner of the vehicle body. The figure demonstrates that the resonance frequencies of the body must be around $2 \mathrm{~Hz}$. The vehicle model, in this case, is a linear 7 degree of freedom (DOF) state-space formulation [12] whose parameters have been revised after various experimental tests on the 4-posters rig. The eigenvalues of the state matrix (with minimum semi-active damping coefficients) are repetitively located at $(-0.3 \pm 10.5)$ and $(-0.8 \pm j 58)$.

The first eigenvalue corresponds to the natural frequency of $\omega_{\mathrm{n}} \approx 1.7 \mathrm{~Hz}$, which matches the sprung mass resonance frequencies. The second one with $w_{n} \approx 9 \mathrm{~Hz}$ represents the resonance frequencies of the wheels. Figure 5 portrays the frequency content of the front left wheel accelerometer signal in response to the 4-poster excitations. It is illustrated that the realresonance frequency of the wheel hub is located at approximately $9 \mathrm{~Hz}$, which is in agreement with the analytical model prediction. The peak at the $2 \mathrm{~Hz}$ belongs to the resonance frequencies 


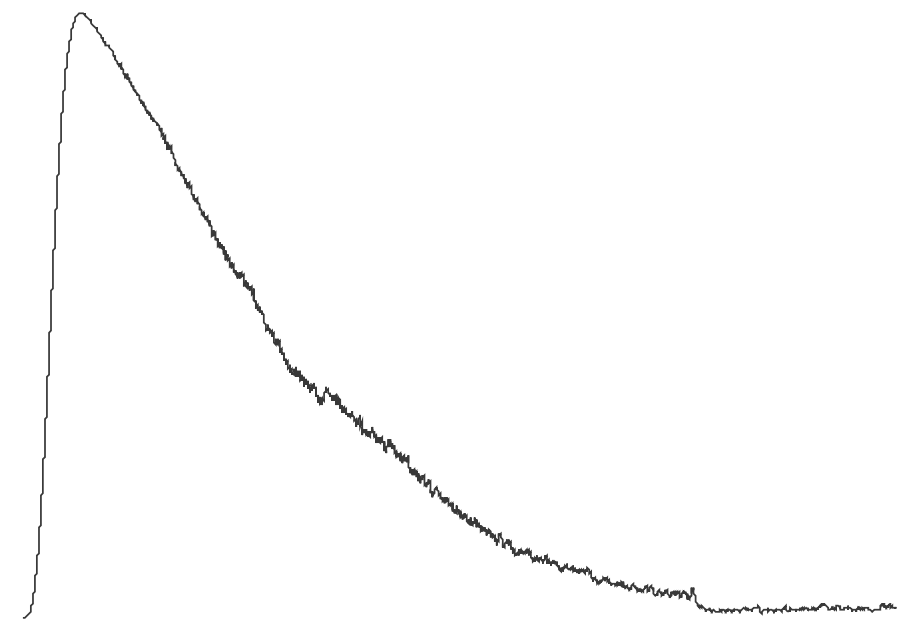

Figure 3. The frequency content of the base shaker input.

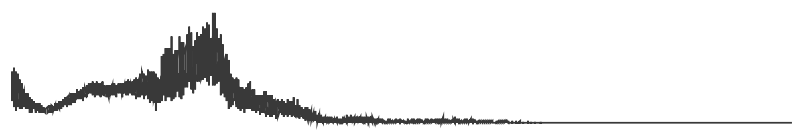

Figure 4. Frequency content of the sprung mass acceleration.

of the body. The other peak at approximately $22 \mathrm{~Hz}$ represents the resonance frequency of a solid component of the front left suspension, which has not been considered in the 7DOF model. To compensate for the inaccuracies and uncertainties in the analytical model, a white noise signal $w(t)$ with a covariance of $Q(t)$ is also considered as an extra input to the model. Matrix $L$ is the noise input matrix in Equation (2). 


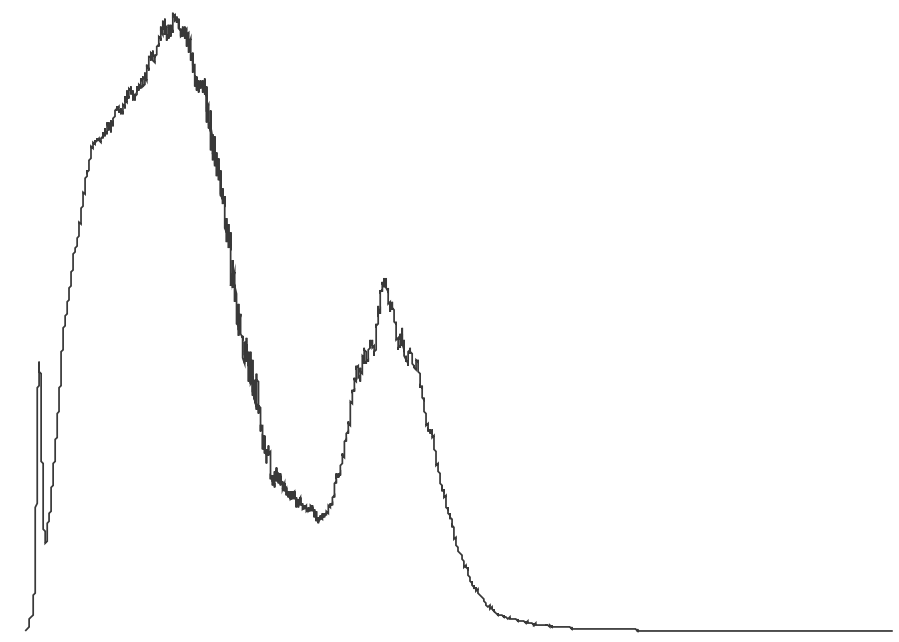

Figure 5. Frequency content of the front left wheel hub acceleration.

Typically, the measurement system model is given as a nonlinear combination of the vehicle states, and the current damping coefficient such that

$$
z=h\left(x, c_{s}\right)+v
$$

The sensors data are assumed to be corrupted by a zero-mean white noise $v$ with a covariance of $R$. By employing a proper sensor configuration, most of the vehicle's required states can be observed through the EKF [12]. In this case, the EKF simply appears as a low-pass filter whose bandwidth is a function of the $Q$ and $R$ matrices. Otherwise, the combination of a low pass filter and a differentiator, or an integrator followed by a high-pass filter, can be utilised to calculate the unobserved states. In either circumstance, the filtering block can be easily substituted by a suitable linear transfer function.

The ultimate control objective is to determine $c_{\min }$ and $c_{\text {max }}$ such that the state vector and its derivatives are minimised $\left(x_{d}=x_{d}^{*}=x_{d}=0\right)$. The state variables that need to be regulated, such as absolute position and velocity of the wheel-hubs, and relative displacement and velocity of the shocks, have frequency components up to $12 \mathrm{~Hz}$, which is the typical resonant frequency of the vehicle wheels. Therefore, the control loop must be performed as fast as at least $60 \mathrm{~Hz}$ ( 5 times faster) to be able to suppress the vibratory motions effectively. Moreover, the closedloop control system must guarantee to meet some given transient response criteria. The lower bound, $\iota_{\min }$, though, is limited to the minimum possible damping coefficient (ideally zero) that the semi-active shock offers (and is determined by the damper characteristic tests). Therefore, the problem is reduced to the determination of $c_{\max }$ so that the desired requirements are satisfied.

The discontinuous block in Figure 1, describing the Skyhook control strategy, is the only module that cannot be classified in the framework of the conventional control system theory. The new approach should bring the discontinuous control strategy into the general framework of the classic control theory, only then can all the conventional control system theory tools can be employed in the design of a semi-active suspension controller. The next section pursues this goal. 


\section{Fuzzy network model of Skyhook}

It can be proven that a Fuzzy logic network is capable of approximating any non-linear function on a compact set to an arbitrary accuracy [13]. Thus, a continuous Fuzzy system can be developed to mimic the on-off Skyhook control strategy and maintain the controller efficiency. One method to create such a network is to encapsulate the Skyhook control logic into the inference engine of the Fuzzy network. This engine is the brain of the Fuzzy system which induces a Fuzzy output, based on a predefined Fuzzy rule base. In this case, the rule base built on the Skyhook strategy consists of the following 4 rules:

$$
\begin{aligned}
& \mathrm{R}^{1}: \mathrm{IF} v_{\mathrm{b}} \text { is 'P' AND } v_{\mathrm{r}} \text { is ' } \mathrm{P} \text { ' THEN } c_{\mathrm{s}} \text { is ' } \mathrm{B} \text { ' } \\
& \mathrm{R}^{2}: \mathrm{IF} v_{\mathrm{b}} \text { is 'N' AND } v_{\mathrm{r}} \text { is ' } \mathrm{P} \text { ' THEN } c_{\mathrm{s}} \text { is ' } \mathrm{S} \text { ' } \\
& \mathrm{R}^{3}: \mathrm{IF} \mathrm{v}_{\mathrm{b}} \text { is 'P' AND } v_{\mathrm{r}} \text { is ' } \mathrm{N} \text { ' THEN } c_{\mathrm{s}} \text { is ' } \mathrm{S} \text { ' } \\
& \mathrm{R}^{4}: \mathrm{IF} \mathrm{v}_{\mathrm{b}} \text { is ' } \mathrm{N} \text { ' AND } v_{\mathrm{r}} \text { is ' } \mathrm{N} \text { ' THEN } c_{\mathrm{s}} \text { is ' } \mathrm{B} \text { ' }
\end{aligned}
$$

where $P$ and $N$ are the primary Fuzzy sets defined in the $R$ which is the universe of discourse of the input variables $\mathrm{v}_{\mathrm{b}}$ and $\mathrm{v}_{\mathrm{r}}$. $\mathrm{P}$ represents 'positive' and $\mathrm{N}$ denotes 'negative', and are selected as follows:

$$
\mu_{p}\left(v_{i}\right)=\frac{k}{1+\exp \left[-v_{i} / q\right]^{\prime}}
$$

and

$$
\|_{N}\left(v_{i}\right)=\frac{k}{1+\exp \left[v_{i} / q\right]}
$$

where $i=b, r \cdot q$ determines the growing or decaying rates of the sigmoid functions. Proper values of $q$ for this particular application are between 0 and $0.1 . k$ is usually set to 1 . The inputs to the Fuzzy system are normalised such that the same membership functions can be employed for both inputs. The normalised crisp input vector $v=\left[\begin{array}{ll}v_{b} & v_{l}\end{array}\right]^{T}$ is then fuzzified via a singleton fuzzifier [13]. Figure 6 shows the basic structure of the Fuzzy network model.

Each IF-THEN rule of the Fuzzy rule base is characterised by a membership function, $\left\|_{R}=\right\|_{R} \times R \times R\left(V, C_{s}\right)$, defined in the Cartesian product space $C=R \times R \times R$. The final structure of the $\|_{R}$, is determined by using a particular operation rule of the Fuzzy implication [13]. By employing the product operation rule, the $\mu_{R}$ is evaluated by

$$
\mu_{R}=\|_{v i}\left(v_{b}\right), \mu_{u}\left(v_{1}\right), \mu_{r} \cdot\left(c_{s}\right)
$$

where $=1, \ldots, 4$ and $j, k$ is either $p$ or $N$, depending the rule number. In addition, $\mu$, is one of the two membership functions defined on the output variable $c_{s}$; that is, 'B' (big) or ' $\mathrm{S}$ ' (small). Then, a Fuzzy output is induced from each IF-THEN rule and the fuzzified input vector $V$ by applying the following equation:

$$
C:=V \circ R,
$$

where ${ }^{\circ}$ refers to a compositional rule of inference. In this design, the sup-product compositional rule is applied $[13,14]$. As a result, $C_{s}$ is evaluated by the following membership

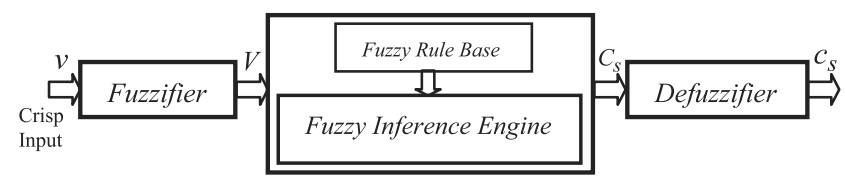

Figure 6. The basic structure of the Fuzzy logic system. 
function

$$
H_{c}:=\sup _{v \in \times R}\left\{\|v(v),\|_{R} \|\left(v, c_{s}\right)\right\}
$$

The output of the Fuzzy inference engine is the union of each individual IF-THEN rule outcome $C$,

$$
C_{\mathrm{s}}={ }_{i=1}^{n=4} c_{\mathrm{s}}
$$

The Fuzzy output set, $C_{s}$, is ultimately transformed into a crisp output by a centre average defuzzifier [13], given by

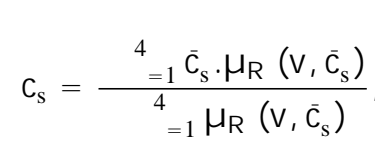

where $c_{s}^{-t}$ is the extremum of the output Fuzzy set, $\mu_{\psi} \|\left(c_{s}\right)$, (at which the $\mu_{\|} \|\left(c_{s}\right)$ has a maximum value of 1). The substitution of Equation (6) in Equation (10) results in the crisp output of the Fuzzy system as:

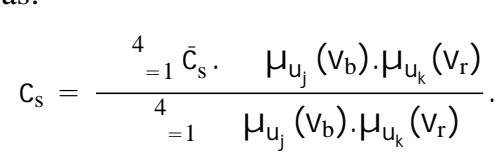

By defining W as

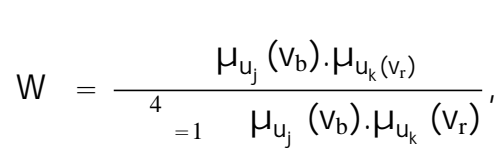

the output of the Fuzzy network is ultimately written in the following compact form:

$$
c_{\mathrm{s}}=\boldsymbol{\gamma}_{i=1}^{\rightarrow} c_{\mathrm{s}} W=W \mathrm{~T}^{\mathrm{T}} \mathrm{C}^{-} \text {. }
$$

In Equation (13), W $W\left[W^{\dagger}\right], c^{-}=\left[c_{\mathrm{s}}^{-\dagger}\right]$, and $c_{\mathrm{s}}^{-\dagger}$ equals either $c_{\max }$ or $c_{\min }$. Having employed the Fuzzy system, the structure of the semi-active suspension control system is illustrated as in Figure 7, where the on-off controller is replaced by the continuous nonlinear map $\mathrm{N}$ defined by Equation (13):

$$
c_{\mathrm{s}}=\mathrm{N}\left(\dot{x}, c_{\max } c_{\min }\right) .
$$

In the next step, the nonlinear control law, Equation (14), is designed to provide some desired performance around a system equilibrium point. In order to do that, $\mathrm{N}$ is expanded around the equilibrium point, $x_{0}$, in which

$$
v_{b_{0}}=v_{r_{0}}=0
$$

After applying the Taylor series expansion formula, the nonlinear function can now be described as follows:

$$
\mathrm{N} \cong \mathrm{N}\left(x_{0}\right)+\frac{\partial \mathrm{N}}{\partial x_{0}} \boldsymbol{8}\left(x-x_{0}\right) .
$$

By the use of Equations (4), (5), and (11), after some mathematical manipulation, it can be shown that

$$
\frac{\partial N}{\partial x_{0}} \frac{8}{x_{0}}=0
$$

due to the symmetry of the membership functions, defined on the inputs, i.e. Equations (4) and (5). Moreover, since $l_{\min }$ is much less than $\varsigma_{\max }$ (zero in theory), the terms containing $\varsigma_{\min }$ 


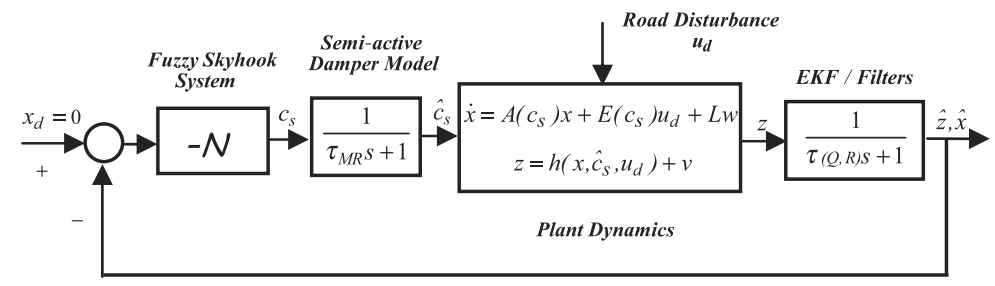

Figure 7. The semi-active suspension control system with the Fuzzy Skyhook system.

are overlooked. And therefore, $\mathbf{N}\left(x_{0}\right)$ is obtained by calculating

$$
\mathrm{N} \cong \mathrm{N}\left(x_{0}\right) \cong \frac{l_{\max }}{r(k, q)}
$$

$r$ is a constant quantity, which relates to the membership function parameters, $k$ and $q$. By properly selecting $k$ and $q, r$ usually falls into a region of $[1.5,2.5]$.

Now, the original switching control system is approximated by a linear state-space model described (around the equilibrium point) by

$$
x_{c}^{*}=A_{c^{X}}{ }_{c}+B c_{c}^{U} c+L c_{c}^{W} c_{1}
$$

where the state, input, and disturbance vectors are given in Equation (20)

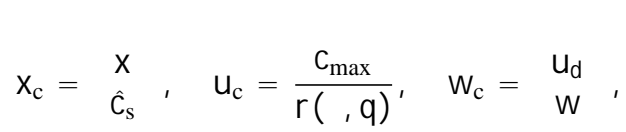

and the corresponding matrices are as follows:

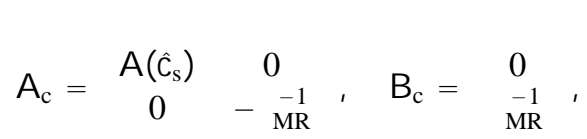

and

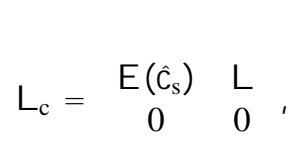

where subscript c refers to the controller design. Subsequently, all the tools, well-known in the conventional control system theory from pole placement, LQR/LQG and $\mathrm{H}_{2 \mid \infty}$ to various frequency domain techniques, can be utilised to design the unknown parameter, $C_{\max }$, such that the closed-loop system satisfies the desired performance requirements in the vicinity of the equilibrium point. For other points of the universe of discourse, the required performance may not be satisfied. However, the exponential stability of the controlled system is always guaranteed in the entire universe of discourse due to the passivity property of the suspension system [15].

THEOREM 1 (Nonlinear Separation Principle) Consider a nonlinear dynamic in the form of $x^{*}=f(x, u),-$ Suppose that $\exists \|=g(x), g|0|=0, g \in\left({ }^{1}\right.$, and $\mathrm{g} g(x)$ is bounded so that the equilibrium point $x_{\text {e }}$ of the $x^{*}=f(x, g(x) \mid$ is exponentially stable. Furthermore, there exists an observer $\hat{x}^{\dot{n}}=h(\hat{x}, z, u)$ which estimates the system state vector such that the estimation error dynamics of the form, $e^{*}=a(e)$, is exponentially stable $\left(e=x^{\wedge}-x\right)$. 
Then $\left[\begin{array}{ll}x_{\mathrm{e}} & 0\end{array}\right]^{\mathrm{T}}$ is an exponentially stable equilibrium point of the integrated system [16],

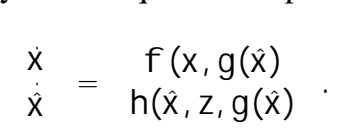

As described in Section 2, an EKF/ filters are separately designed [12] (with the proper bandwidth) which provides the vehicle states. Theorem 1 confirms that the integrated estimator and controller loop of Figure 7, which is described by the following equation:

$$
\dot{x}_{s}^{*}=f_{s}\left(x, \hat{x}, z, c_{\max } w\right),
$$

is exponentially stable in the entire universe of discourse, and guarantees the desired performance in the vicinity of the equilibrium point $\left[\begin{array}{ll}x_{0} & 0\end{array}\right]^{\mathrm{T}}$. The concatenated state vector in Equation (22) is

$$
x_{s}=\begin{aligned}
& x^{\wedge} \\
& \hat{n}_{s}
\end{aligned}
$$

where subscript $\mathrm{s}$ refers to the semi-active closed-loop control system.

In practice, either the original bang-bang Skyhook strategy, Equation (1), or the nonlinear Fuzzy controller (14) is implemented by incorporating the designed $c_{\max }$. However, $c_{\max }$, obtained by the proposed methodology, might need to be fine-tuned during road tests to assure the desired performance. In the case of the Fuzzy controller, the outcome (Equation (13) or (14)) is a simple continuous representation of the original Skyhook control strategy with a few lines of code that is simply embedded in a low-cost micro controller. By tuning the membership function parameters (see Equations (4) and (5)), sensitivity and smoothness of the Fuzzy Skyhook controller can also be altered and adjusted. q changes the membership function growth or decay rate. Setting q to small quantities, increases the sharpness of the Fuzzy controller; that is, it behaves more or less similar to the original on-off controller. Moreover, to decrease sensitivity of the Fuzzy controller to the input noises, an offset value $\gamma$ is introduced to the input membership functions as follows:

$$
\mu_{p}\left(v_{i}\right)=\frac{k}{1+\exp \left[-\left(v_{i}-v\right) / q\right]}
$$

and,

$$
\|_{N}\left(v_{i}\right)=\frac{k}{1+\exp \left[\left(v_{i}+v\right) / q\right]}
$$

The greater the $\gamma$ is selected, the less sensitive the controller becomes to the input variables $v=\left[\begin{array}{lll}v_{b} & v_{r}\end{array}\right]^{T}$ noise.

The design procedure is summarised in the following six steps:

(1) Establish the semi-active control system desired performance.

(2) Find out the vehicle information such as mass, moment of inertia, and dimensions. Then, develop the vehicle's vertical model state-space realisation (see Equation (2)), accordingly.

(3) Determine and adjust the parameters of the input Fuzzy sets (see Equations (4) and (5)). Then, calculate $r(k, q)$ in Equation (18). As pointed out earlier, proper $k$ and $q$ result in a number between 1.5 and 2.5 for $\uparrow$. Such an outcome leads to a convenient rule-ofthumb expression for choosing, 1 , which bypasses the entire mathematical manipulations required in this step. As a rule-of-thumb, r can be selected in the [1.5, 2.5] region; r $=1.5$ results in a smoother, controller and softer suspension (luxury vehicles), whereas ${ }^{\mathrm{I}}=2.5$ offers a harder suspension (sporty vehicles). 
(4) Consider the approximated controlled-system realisation, given by Equations (19)-(21). By using a well-known time domain/ frequency domain design technique, extract $c_{\max }$ such that eigenvalues of corresponding state matrix $A_{c}$ are placed in the proper regions of the $s$-plane.

(5) With $c_{\max }$, determined from the previous step, implement either the on-off Skyhook strategy (Equation (1)) or the nonlinear Fuzzy controller (Equation (13)).

(6) Fine-tune the $c_{\max }$ ballpark estimate during road tests to guarantee the desired ride and stability requirements.

Effectiveness of the proposed design methodology is then demonstrated in the next section through design, implementation, and tests of a semi-active control system for a Cadillac SRX 2005 .

\section{Case study and real-time results}

To demonstrate the effectiveness of the proposed methodology, design of a semi-active suspension system for the Cadillac SRX model 2005 is investigated. Some of the Cadillac SRX specifications are listed in Table 1 [17].

The test vehicle is equipped with MagneRide ${ }^{\mathrm{TM}}$ semi-active MR dampers. The dampers are tested in the University of Waterloo's MTS lab to characterise the damping forces with respect to the applied control current and the relative velocity across the shock (Figure 8).

Figure 9 plots the rear dampers test results for different supplied currents. It is experimentally shown that the damper is capable of providing a wide range of damping coefficients from $200 \mathrm{~N} \mathrm{sec} / \mathrm{m}$ at $0 \mathrm{Amp}$ to about approximately $7000 \mathrm{~N} \mathrm{sec/m}$ at $5 \mathrm{Amp}$.

A semi-active control problem is now defined as follows. It is desirable to find appropriate lower and upper bounds of the Skyhook control strategy (or the hybrid strategy), that is, $l_{\mathrm{min}}$ and $c_{\max }$, such that the following performance requirements are fulfilled

Table 1. Cadillac SRX exterior dimensions.

\begin{tabular}{lc}
\hline Wheelbase $(\mathrm{mm})$ & 2957 \\
Overall length $(\mathrm{mm})$ & 4950 \\
Overall width $(\mathrm{mm})$ & 1844 \\
Overall height $(\mathrm{mm})$ & 1722 \\
Track $(\mathrm{mm})$ & Front: 2957, Rear: 1580 \\
Base curb weight $(\mathrm{kg})$ & 2013 \\
Weight distribution $(\%$ front/ rear) & $52 / 48$ \\
\hline
\end{tabular}

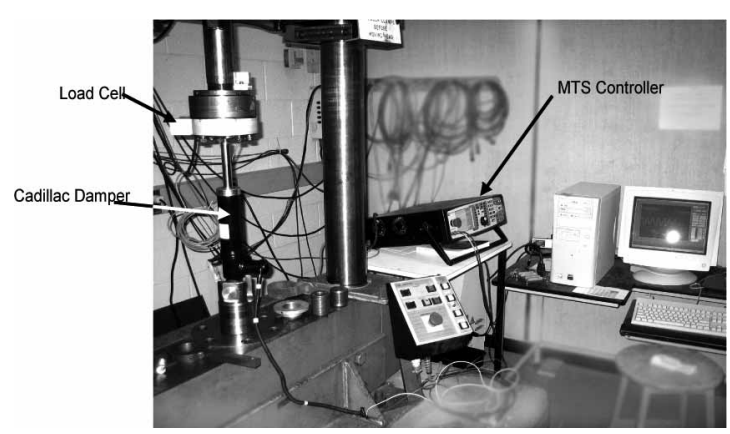

Figure 8. The MTS test setup of the University of Waterloo. 


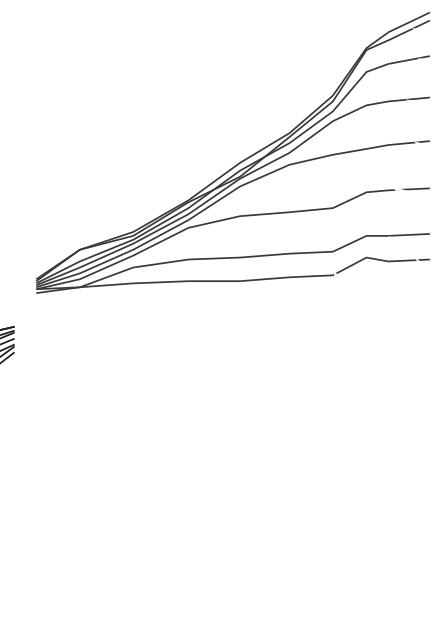

Figure 9. Cadillac SRX MR damper behaviour.

- soft ride on even roads/ pavement (luxury style),

- controlled ride on rough roads/ bumps with a settling time of around $1 \mathrm{sec}$ and a damping ratio of approximately $\}=0.4$ (not more than 2 bounces after the disturbance).

Note that the design requirements are subjective and vary from one designer to the next.

The vehicle vertical model is first derived [12] and verified through 4-poster tests, as described in Section 2. The test vehicle is also instrumented with a full distributed sensor configuration consisting of eight accelerometers, one inertial measurement unit (IMU) and four displacement sensors.

The displacement sensors $[18,19]$, installed by the vehicle manufacturer, measure the relative displacement across the shock, as shown in Figure 10. As introduced by Equation (1), the Skyhook control strategy requires information regarding the sprung mass motion, as well as its relative motion with respect to the wheels. Therefore, two accelerometers are installed at the ends of each shock, one at the body end and the other close to each wheel hub, totalling eight. Figure 11 illustrates an accelerometer [20,21], mounted near the rear left wheel hub. The IMU [21,22] is mounted in the vicinity of the CG to measure the vehicle's body 6DOF motion.

In the next step, EKF/ filters are developed [12] to fuse the measurements of the sensors and estimate the vehicle states required by the semi-active controller.

By considering the design requirements, the membership function (see Equations (4) and (5)) parameters are chosen as follows:

$$
k=1, \quad q=0.01, \quad y=0.05 \text {. }
$$

As mentioned before, $k$ is usually selected to be one. With $q$ set to the small quantity of 0.01 , the Fuzzy controller behaves similarly to the on-off Skyhook controller. $\gamma=0.05$ renders the Fuzzy controller insensitive to low-amplitude vibrations of the vehicle's body and wheels, caused by weak disturbances. And consequently, the controller maintains the damping of the MR dampers at the most minimum values (around $c_{\min }$ ) when the vehicle is moving on the 

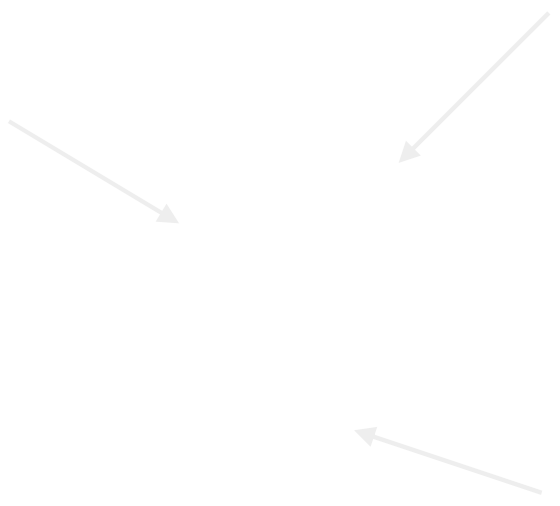

Figure 10. The Cadillac SRX suspension compartment.

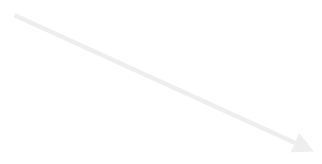

Figure 11. The accelerometer mounted at the wheel end of the MR damper.

even segments of a road. This results in a luxury style comfortable ride. From the known parameters of Equation (23), $r$ is then calculated to be 2.2.

The next step considers the approximated controlled system (Equations (19)-(21)) and the given I to determine a proper maximum limit for the damping coefficient, $c_{\max }$. The desired closed-loop poles (or regions in the $s$-plane) are first specified in accordance with the performance requirements. Then, by using the pole placement technique (or any other similar design methodology), $I_{\max }$ is calculated to satisfy the requirements. For this particular semi-active control problem, $C_{\max }$ is obtained to be around $5500 \mathrm{~N} \mathrm{sec} / \mathrm{m}$ From the MR damper characteristic equations, it is revealed that the required upper damping is achieved at approximately 4 Amp of the supplied current. However, the lower bound of the damping coefficient, ${ }{ }_{\min }$, is obtained by the damper characteristic tests and is approximately $200 \mathrm{~N} \mathrm{sec} / \mathrm{m}$ at $0.0 \mathrm{Amp}$ of the supplied current.

The last is investigation of the efficiency of the designed semi-active suspension system by conducting experiments. A real-time processing platform, running in $\mathrm{VC}++$, is developed to implement the integrated estimator and controller. A Pentium 4 desktop computer with 3.4 GHz computational power and $1 \mathrm{~GB}$ instant memory is adopted as the onboard processing machine. The sensors data are collected at $500 \mathrm{~Hz}$ from the accelerometers and IMU, and approximately $40 \mathrm{~Hz}$ from the displacement sensors, and fed to the estimator. The computed 
control command is sent at the rate of around $20 \mathrm{~Hz}$ (Theoretically, it must be at least $60 \mathrm{~Hz}$ to effectively suppress the fast frequency components of the vehicle vibrations. However, due to the limitation of the MR damper bandwidth (approximately $10 \mathrm{~Hz}$ ), the controller command is sent at a rate of $20 \mathrm{~Hz}$ ) out of the computer through an RS485 serial port to a voltage-controlled current circuit. The circuit drives a PWM amplifier, which accordingly, feeds the MR dampers. The road tests are carried out on the University of Waterloo's ring road where there is a bump, as shown in Figure 12.

The test vehicle is driven on a specified section of the ring road (including the bump) with speeds between 20 and $50 \mathrm{~km} / \mathrm{h}$. For each velocity, the performance of the Cadillac SRX original controller is compared with that of the designed controller. The measurements from the vertical accelerometer of the IMU are used as the ride comfort measure. Figure 13 reflects the acceleration of the CG for two cases: when the benchmark control system is engaged and when the integrated estimator and Fuzzy Skyhook controller is in-line. In comparison with the Cadillac controller, the Fuzzy Skyhook controller decreases the maximum acceleration of the vehicle's CG and the peak to peak value by $19 \%$ and $13 \%$, respectively.

Figure 12. The bump in the UW ring road used for the field tests.

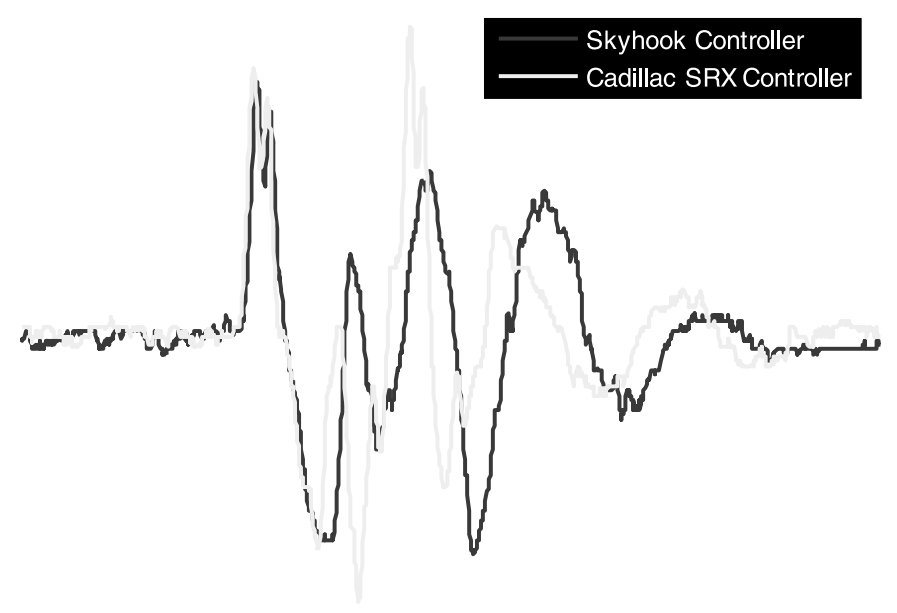

Figure 13. The graph compares the performance of the integrated system and the Cadillac SRX original controller (the test vehicle speed is $30 \mathrm{~km} / \mathrm{h}$ ). 


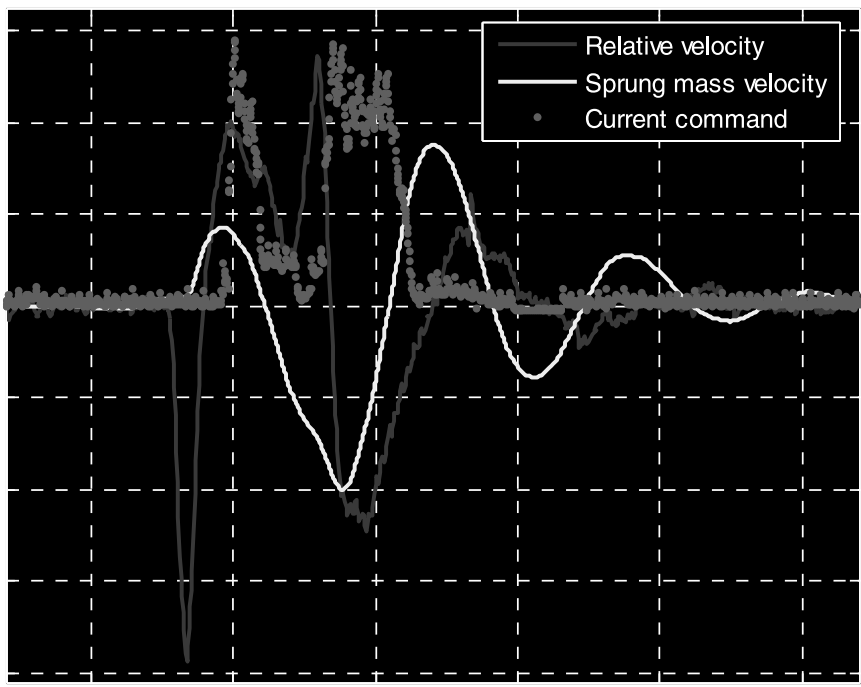

Figure 14. Absolute velocity of the front left corner of the body, relative velocity of the associated MR damper and the control current sent to the damper.

The settling time of the closed-loop system is around $1.5 \mathrm{~s}$ (the bump input ends at approximately $t=85 \mathrm{~s}$ ), which is close to the desired magnitude.

Figure 14 puts the command signal, sent by the Fuzzy Skyhook controller, and the controller input $\left(V=\left[\begin{array}{ll}V_{b} & V_{l}\end{array}\right]^{\mathrm{T}}\right)$ signals, side by side. It is apparent that the designed controller is not sensitive to small perturbations. The low-damping value, assigned by the controller on the even segments of the pavement provides a soft and comfortable ride. Also, the controller effectively acts to reduce the effects of the bump, as seen in Figures 13 and 14. The entire processing time is evaluated as less than $2 \mathrm{~ms}$, which indicates that the integrated estimator and controller software can be implemented by the use of a single low-cost micro-controller.

Overall, it is observed that employing the systematic design methodology, proposed in this paper, results in a semi-active suspension system whose performance is comparable with that of a commercialised industrial system. Also, it is confirmed that the proposed technique makes the semi-active control system design straightforward and free from the need of numerous trial-and-error attempts. Small modifications on the Fuzzy controller performance can be achieved by altering and adjusting the membership function parameters. However, to satisfy different performance requirements entirely (different settling time and transient response requirements), the design steps should be repeated to generate new values of $c_{\max }$ and $c_{\min }$.

\section{Sub-optimal Skyhook control strategy}

Figure 15 shows the relative displacement and velocity of the front left damper, along with the absolute velocity of the sprung mass. The data are generated through the computer simulation of the 7DOF Cadillac model (Equation (2)) passing over the same bump at $40 \mathrm{~km} / \mathrm{h}$. From this graph and similar simulation results for other speeds, it is discovered that the behaviour of the sign change of the $v_{r}, v_{b}$ (relative velocity times the absolute velocity) is opposite to that of the $v_{r}, z_{r}$ (relative velocity and relative displacement product) [23]. This behaviour is further clarified in Figure 15, where the non-zero status of the green line refers to the positive 


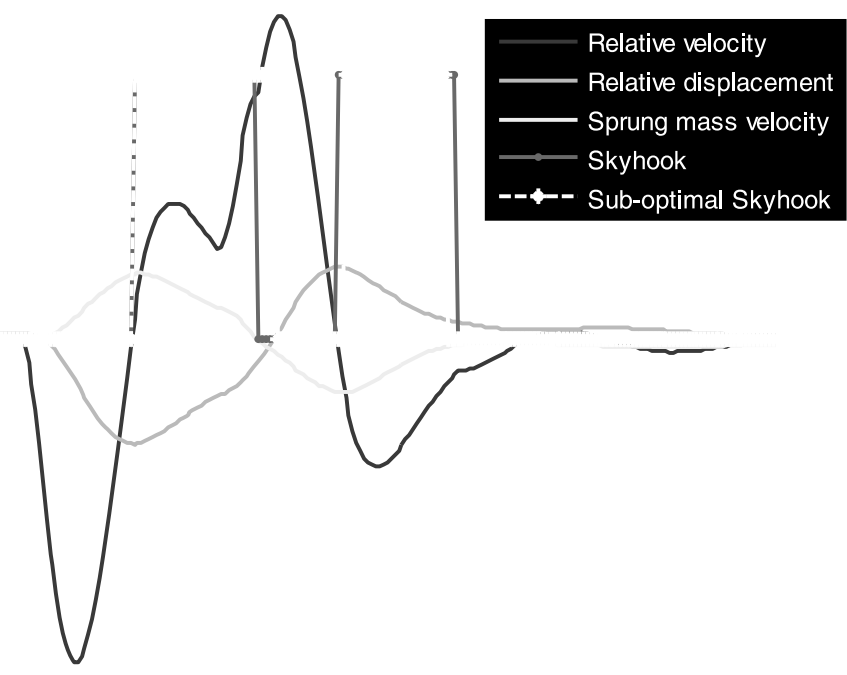

Figure 15. The graph compares signs of the relative and absolute velocity product and the relative velocity and displacement product.

sign of the relative and absolute velocity product, while the non-zero status of the black curve refers to the negative sign of the relative velocity and the relative displacement product. It is apparent that the two curves almost overlap, which means that when the relative velocity times the absolute velocity is positive, the relative velocity times the relative displacement is negative.

Figure 15, or similar simulation results, implies that the sign of the product of the relative velocity and the relative displacement can be monitored as a replacement for the original Skyhook controller switching criterion. The proposed alternative eliminates the need for computing the sprung mass absolute velocity. However, the performance of the semi-active control system is lowered to some extent. The sub-optimal Skyhook control policy is then given by [23]:

$$
c_{\text {sub_sky }}=\begin{array}{ll}
l_{\max } & \text { if } v_{\mathrm{r}}{ }_{\mathrm{r}} \leq 0 \\
l_{\text {min }} & \text { otherwise }
\end{array}
$$

where $l_{\mathrm{r}}$ denotes the relative displacement across the shock.

To investigate the effectiveness of the proposed design methodology, another semi-active control problem with different design requirements and the sub-optimal Skyhook control strategy is studied next. Consider the design requirements of the previous semi-active control problem except that the desired damping ratio is decreased by $10 \%$. Similar to Section 4 , by following the step by step design procedure, the upper damping limit of the MR dampers, $C$ max , is calculated to be around $5000 \mathrm{~N} \mathrm{sec} / \mathrm{m}$ (at approximately $3 \mathrm{Amp}$ of the supplied current). The control strategy, given in Equation (24), with the calculated $c_{\max }$, is directly coded to the embedded processor. Experiments are carried out with the same vehicle, manoeuvres, and the road conditions. Figure 16 offers a comparison of the performance of the integrated estimator and the sub-optimal Skyhook controller and the Cadillac SRX original controller. It is illustrated that the suspension system with the sub-optimal Skyhook controller performs in a similar manner to the Cadillac SRX original semi-active control system. 


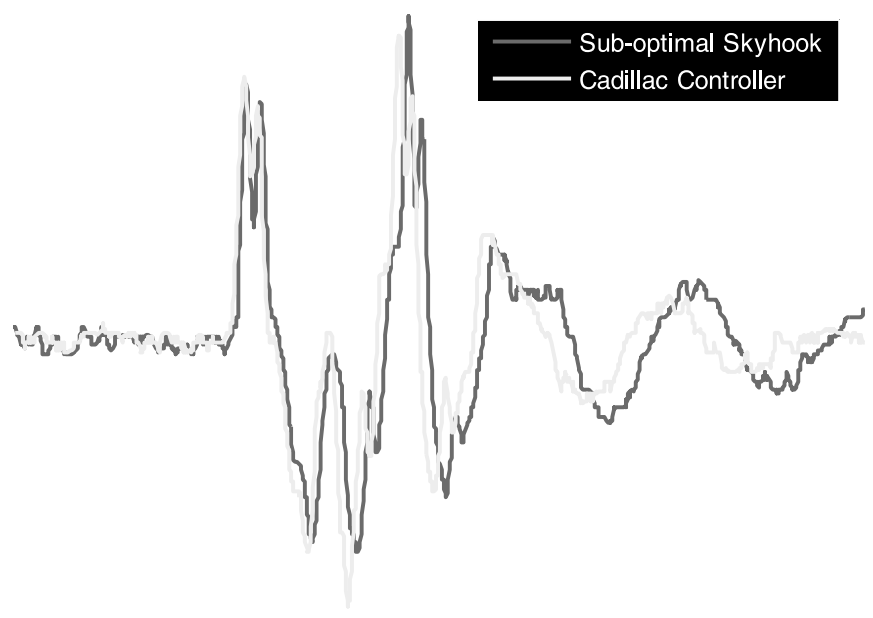

Figure 16. The graph compares the performance of the sub-optimal Skyhook controller with the Cadillac SRX semi-active controller.

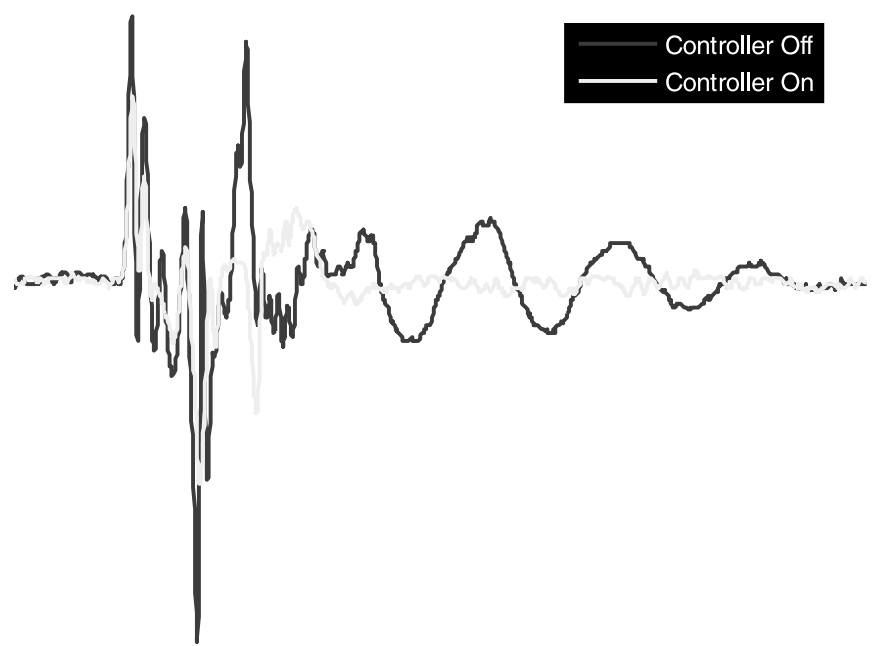

Figure 17. Performance of the sub-optimal Skyhook suspension system.

To demonstrate the efficiency of the integrated control system further, Figure 17 plots the accelerations of the vehicle CG for two situations: when the controller is engaged and when it is off. The vehicle is driven on the same road/ bump but with a different speed of $40 \mathrm{~km} / \mathrm{h}$. It is obvious that when the suspension controller is in-line, the transmitted disturbance is significantly reduced. 


\section{Conclusion}

In this paper, a systematic while practical methodology is devised for the design of vehicle semi-active suspension systems. The proposed methodology maps the semi-active control problem to the linear region, where all the well-known conventional design techniques can be implemented. Once the controller parameters are determined in the linear region, an inverse mapping offers the control law. The effectiveness of the proposed methodology is illustrated through the design of a semi-active suspension system for a Cadillac SRX 2005. The road test results confirm that following the systematic design steps results in a semi-active suspension system whose performance is comparable with that of a Cadillac SRX suspension system. It is also demonstrated that the design process becomes straightforward and free of numerous iterations.

\section{Acknowledgements}

The authors would like to thank the Phoenix research team at the University of Waterloo and Simon Fraser University. Also, the financial support, provided by Mechworks Systems Inc. and Ontario Centres of Excellence, is greatly appreciated.

\section{References}

[1] D. Karnopp, M.J. Crosby, and R.A. Harwood, Vibration control using semi-active force generators, ASME J. Eng. Ind. 2 (1974), pp. 619-626.

[2] M. Novak and M. Valasek, A new concept of semi-active control of trucks suspension, in Proceedings of AVEC 96, International Symposium on Advanced Vehicle Control, Aachen University of Technology, 1996, pp. 141-151.

[3] K. Hong, H. Sohn, and J.K. Hedrick, Modified Skyhook control of semi-active suspensions: A new model, gain scheduling, and hardware in the loop tuning, ASME J. Dyn. Syst. Meas. Control 124 (2002), pp. 158-167.

[4] Y. Liu, F. Gordaninejad, C. Evrensel, S. Karakas, and U. Dogruer, Experimental study on Fuzzy skyhook control of a vehicle suspension system using a magneto-rheological fluid damper, in Proceedings of SPIE, Smart Structure and Material: Industrial and Commercial Applications of Smart Structure Technologies Vol. 5388, 2004, pp. 338-347.

[5] M. Ahmadian, F.D. Goncalves, and C. Sandu, An experimental analysis of suitability of various semi-active control methods for magneto-rheological vehicle suspensions, in Proceedings of SPIE, Smart Structure and Material: Damping and Isolation Vol. 5760, 2005, pp. 208-222.

[6] M. Ahmadian, On the development of Fuzzy skyhook control for semi-active magneto rheological systems, in Proceedings of SPIE, Smart Structure and Material: Damping and Isolation Vol. 5760, 2005, pp. 268-282.

[7] J. Lieh and W.J. Li, Adaptive Fuzzy control of vehicle semi-active suspension control, ASME J. Dyn. Syst. Meas. Control 61 (2004), pp. 293-297.

[8] D.E. Ivers and L.R. Miller, Experimental comparison of passive, semi-active on| off, and semi-active continuous suspensions, SAE Technical Paper Series No. 892484, 1989.

[9] D. Sammier, O. Sename, and L. Dugard, Skyhook and $H_{\infty}$ control of semi-active suspensions: Some practical aspects, Veh. Syst. Dyn. 39(4) (2003), pp. 279-308.

[10] F.D. Goncalves, J. Koo, and M. Ahmadian, Experimental approach for finding the response time of MR dampers for vehicles applications, in Proceedings of DETC'03: ASME 2003 Design Engineering Technical Conferences and Computers and Information in Engineering Conference. Chicago, Illlinois USA, 2003.

[11] Delphi Corporation, The Delphi MagneRide ${ }^{\mathrm{TM}}$ semi-active shock absorber. Available at http:| / www.delphi.com.

[12] H. Bolandhemmat, C. Clark, and F. Golnaraghi, A Distributed Sensing System for Vehicles States Estimation, Proceedings of IMECE2006: ASME International Mechanical Engineering Congress and Exposition, Chicago, Illinois, USA, 2006.

[13] L.-X. Wang, A Course in Fuzzy Systems and Control, 2nd ed., New York, Prentice Hall, 1997.

[14] L.A. Zadeh, Outline of a new approach to the analysis of complex systems and decision processes, IEEE Trans. Syst. Man Cybern. 3(1) (1973), pp. 28-44.

[15] H.K. Khalil, Nonlinear Systems, 3rd ed., Prentice Hall, 2002.

[16] M. Vidyasagar, On estimation of nonlinear systems using state detection, IEEE Trans. Autom. Control 25 (1980), pp. 504-510.

[17] SRX Standard and Optional Features; software available at http:// gmcanada.com.

[18] Wabash Technologies, 2003. Available at http:/ / www.wabashtech.com. 
[19] Delphi Non-Contact Rotary Position Sensor, 2008. Available at http:// www.delphi.com/ shared/ pdf/ppd/ sensors| ch_rotarypos.pdf.

[20] Analog Devices Specification Manual for ADXL202E Accelerometer and ADXRS401 Single Chip Rate, Gyro, 2000. Available at http:/ / analog.com.

[21] Mechworks Systems Inc, MechSense ${ }^{\mathrm{TM}}$ MD S 202U Accelerometer data sheet, 2004. Available at http:/ / www. mechwokssys.com.

[22] Mechworks Systems Inc, Mech Track ${ }^{\mathrm{TM}}$ IMU data sheet, 2004. Available at http:| / www.mechwokssys.com.

[23] S. Rakheja and S. Sankar, Vibration and shock isolation performance of a semi-active on-off damper, J. Vib. Acoust. Stress Reliab. Design 107(4) (1985), pp. 398-403. 